\section{Sobrepeso e obesidade abdominal em adultos quilombolas, Bahia, Brasil}

\author{
Overweight and abdominal obesity in adults in \\ a quilombo community in Bahia State, Brazil
}

\author{
El sobrepeso y la obesidad abdominal en adultos \\ quilombolas, Bahía, Brasil
}

\author{
${ }^{1}$ Instituto Multidisciplinar \\ em Saúde, Universidade \\ Federal da Bahia, Salvador, \\ Brasil. \\ 2 Faculdade de Medicina \\ Universidade Federal de \\ Minas Gerais, Belo Horizonte, \\ Brasil. \\ Correspondência \\ D. A. Soares \\ Instituto Multidisciplinar em \\ Saúde, Universidade Federal \\ da Bahia. \\ Rua Rio de Contas 58, \\ Quadra 17, Lote 58, Vitória \\ da Conquista, $B A$ \\ 45029-094, Brasil. \\ dandani23@yahoo.com.br
}

\begin{abstract}
This study analyzes nutritional status, estimates the prevalence of overweight and abdominal obesity, and investigates factors associated with these outcomes in a two-stage random sample of adults (> 20 years) in quilombos (communities that descend from African slaves) in Vitória da Conquista, Bahia State, Brazil, in 2011. Among 739 participants, prevalence rates were $31.8 \%$ and $10.2 \%$ for overweight and obesity, respectively, and 55.7\% for increased waist-to-height ratio (>0.50). Prevalence of overweight was higher among 30-39-year-olds, while abdominal obesity was more frequent among older individuals. Female sex, eating chicken or beef with untrimmed fat, and hypertension were associated with higher odds of overweight and abdominal obesity, while smoking and single marital status were associated with lower odds. The results show high prevalence rates for overweight and abdominal obesity in these very poor and socially isolated communities. Specific preventive and control measures are urgently needed.
\end{abstract}

Overweight; Abdominal Obesity; Body Height; African Continental Ancestry Group
Daniela Arruda Soares 1 Sandhi Maria Barreto ${ }^{2}$

\section{Resumo}

Este estudo caracteriza o estado nutricional, estima a prevalência de sobrepeso e de obesidade abdominal e investiga os fatores associados em adultos de comunidades quilombolas. Amostra aleatória em dois estágios, composta de 739 adultos com mais de 20 anos, residentes em comunidades quilombolas, em Vitória da Conquista, Bahia, Brasil, 2011. As prevalências de sobrepeso e de obesidade corporal foram, respectivamente, 31,8\% e 10,2\%, enquanto a de razão cintura-estatura aumentada (>0,50) foi 55,7\%. Sexo feminino, consumo de frango ou carne com gordura aparente e hipertensão aumentaram a chance para os dois indicadores, ao passo que tabagismo e estado civil não casado diminuíram essa chance. O sobrepeso foi maior na faixa etária de 30 a 39 anos, e a obesidade abdominal foi mais frequente em idades mais velhas. Ter baixa escolaridade e não trabalhar foram fatores que elevaram a chance de obesidade abdominal. As prevalências desses eventos são elevadas em uma população de alta vulnerabilidade social, indicando a necessidade de ações específicas de prevenção e controle da obesidade nessas comunidades.

Sobrepeso; Obesidade Abdominal; Estatura; Grupo com Ancestrais do Continente Africano 


\section{Introdução}

As mudanças epidemiológicas, demográficas e nutricionais em curso no Brasil e em outros países emergentes caracterizam-se por decréscimo nos níveis de morbimortalidade e fecundidade, aumento da expectativa de vida, modificações no estilo de vida e nos hábitos alimentares 1,2,3, além de crescimento da carga de doenças crônicas não transmissíveis (DCNT). Tais mudanças afetam toda a população, mas seu impacto entre minorias étnicas e raciais 4,5 no Brasil, em especial em comunidades quilombolas 6,7 , é pouco conhecido. Estudos de base populacional, como a Pesquisa de Orçamentos Familiares (POF) 8, não possibilitam a desagregação de informações para essas populações.

A população afrodescendente foi praticamente transplantada para o Brasil, sendo os quilombos uma forma de organização social contra a opressão sofrida no período de escravidão, o qual perdurou por mais de 300 anos 9. Essa trajetória repercutiu no acesso diferenciado dessa população a bens e serviços 10, afetando o seu modo de vida, padrões de alimentação e, consequentemente, o seu adoecimento e morte 6,7,11,12. O contato de populações tradicionais com a sociedade nacional exerce particular influência sobre seu estado nutricional 7,13,14,15, tendo contribuído tanto para o aparecimento de distúrbios nutricionais carenciais, quanto para o sobrepeso e a obesidade.

As comunidades quilombolas são agrupamentos étnicos que se autodefinem por meio das relações com a terra, o parentesco, o território, a ancestralidade e práticas culturais próprias 16 , representando o principal símbolo de resistência ao regime escravocrata no país. A Região Nordeste conta com o maior número de comunidades quilombolas certificadas ${ }^{17}$. Geralmente, elas se localizam no semiárido, sendo integrantes do polígono das secas, onde ocorrem periodicamente secas anômalas, que geram catástrofes sociais, econômicas e nutricionais frequentes. As atividades econômicas predominantes são a agricultura de subsistência, a pecuária tradicional e o artesanato. Embora os territórios ancestrais configurem importante elemento de identidade grupal 7,9, a sazonalidade climática, a devastação ambiental, as dificuldades de titulação fundiária e de acesso podem contribuir para um risco nutricional diferenciado 15 .

O objetivo deste estudo foi caracterizar o estado nutricional, estimar a prevalência de sobrepeso e obesidade abdominal, assim como os fatores associados a estes, de adultos residentes em comunidades quilombolas na cidade de Vitória da Conquista, Bahia, Brasil.

\section{Materiais e métodos}

\section{Delineamento do estudo}

O presente trabalho insere-se no Projeto COMQUISTA: Comunidades Quilombolas de Vitória da Conquista - Avaliação de Saúde e seus Condicionantes, um estudo de corte transversal realizado em 2011. Originalmente realizado com indivíduos adultos, com 18 anos ou mais, o estudo objetivou investigar características socioeconômicas e demográficas, além de indicadores das condições de vida e saúde e de utilização dos serviços de saúde dessa população.

\section{População de estudo}

O Município de Vitória da Conquista conta com 25 comunidades quilombolas reconhecidas legalmente, localizadas em cinco distritos da região, os quais distam $31,2 \mathrm{~km}$ da sede da cidade (Figura 1). Os africanos que vieram para a Bahia eram predominantemente oriundos de Gana, Nigéria, Benin, Angola, República Democrática do Congo, Moçambique e, em menor número, do Sudão, Costa do Marfim e Chade 18. O acesso a muitas dessas comunidades é difícil devido ao estado de conservação das estradas e às características geográficas, marcadas por morros e ladeiras.

\section{Plano amostral}

A população-alvo do Projeto COMQUISTA foi estimada em 2.935 indivíduos provenientes das dez comunidades sediadas em cinco distritos da região, conforme informações da Secretária Municipal de Saúde e Fundação Palmares. Esse quantitativo corresponde a uma amostra representativa da população quilombola residente na zona rural de Vitória da Conquista. O cálculo amostral considerou uma prevalência de 50\%, precisão de $5 \%$, intervalo de $95 \%$ de confiança (IC95\%), efeito de desenho $=2$ e $30 \%$ de perdas, totalizando 884 indivíduos. A seleção amostral foi realizada em dois estágios. No primeiro, uma comunidade foi selecionada aleatoriamente por distrito, com probabilidade proporcional ao número de habitantes que nela viviam, totalizando cinco comunidades (Corta Lote, Maria Clemência, Furadinho, Lagoa de Melquíades e Boqueirão). Estas obedeceram aos seguintes critérios de inclusão: estarem certificadas pela Fundação Palmares e, por questões operacionais, contarem com o mínimo de cinquenta famílias. No segundo estágio, foi feita seleção dos domicílios de acordo com a distribuição proporcional destes por distrito. Para essa seleção, foi utilizado um 
Figura 1

Mapa das comunidades quilombolas selecionadas. Projeto COMQUISTA, Vitória da Conquista, Bahia, Brasil, 2011.



Fonte: Comunidades quilombolas: mapeamento Projeto COMQUISTA. 
sistema de georreferenciamento por GPS, com posterior construção de mapas geográficos das comunidades e fotografias dos domicílios, a fim de facilitar a sua localização. Com base na lista de domicílios obtida por comunidade, foi realizado sorteio aleatório sem repetição.

Foram convidados a participar todos os indivíduos que residiam nos domicílios selecionados e que concordaram em assinar o Termo de Consentimento Livre e Esclarecido.

\section{Coleta de dados}

O período de coleta compreendeu os meses de setembro a outubro de 2011, após a condução de um estudo-piloto para se testar a aplicabilidade do instrumento e a logística do campo. Os dados foram coletados por meio de entrevistas individuais, utilizando-se computadores portáteis (HP Pocket Rx5710, Hewlett-Packard Development Company, Estados Unidos). Posteriormente, as informações foram transferidas para um banco de dados próprio, no qual foram armazenadas, minimizando possíveis erros de digitação e facilitando a conferência de eventuais inconsistências. O instrumento utilizado na entrevista foi o questionário semiestruturado da Pesquisa Nacional de Saúde (PNS), o qual passou por etapas de avaliação, pré-teste e adequações pela equipe do projeto. Inicialmente, o questionário foi avaliado com a finalidade de adaptar o vocabulário e algumas perguntas à população e ao contexto em que seria aplicado. Para ajustar o questionário antes da sua aplicação em estudo-piloto, a linguagem, sequência, coerência entre as questões, bem como o tempo necessário para aplicação das entrevistas, foram avaliados em pré-testes. Após o estudo-piloto, algumas informações que seriam investigadas pelo módulo domiciliar foram incluídas no módulo individual, como a relação de parentesco entre os moradores, rendimentos, cobertura de plano de saúde e utilização de serviços de saúde.

As medidas antropométricas e de pressão arterial foram realizadas por entrevistadores treinados, seguindo protocolos padronizados. A aferição da pressão arterial foi feita com esfigmomanômetro digital da marca Omron, modelo HEM-742 (OMRON Corp., São Paulo, Brasil), sendo tomadas três medidas de pressão arterial para cada indivíduo. Para mensuração do peso, foi utilizada balança eletrônica portátil da marca Marte, modelo LC200PP (Marte, São Paulo, Brasil), com capacidade para $200 \mathrm{~kg}$ e sensibilidade de 50g, estando os indivíduos descalços, com o mínimo de roupas e adereços. A altura foi aferida na posição ortostática, braços estendidos ao longo do corpo, cabeça erguida, sendo utilizado um estadiômetro portátil, marca CauMaq, modelo est-22, com capacidade para $300 \mathrm{~mm}$ a $2000 \mathrm{~mm}$, precisão de $0,1 \mathrm{~cm}$ (CauMaq Indústria Metalúrgica Ltda., Cachoeira do Sul, Brasil). A circunferência da cintura foi mensurada no ponto médio, situado entre a crista ilíaca e o último arco costal, com fita métrica inelástica da marca Cardiomed, modelo T-87-Wiso, capacidade de $2 \mathrm{~m}$ e precisão de $0,1 \mathrm{~cm}$. As medidas antropométricas 19 e a aferição de pressão arterial 20 seguiram técnicas previamente estabelecidas. O controle de qualidade do estudo foi realizado mediante segunda entrevista com cerca de $5 \%$ da amostra de cada comunidade e aplicação do questionário na íntegra. O teste kappa, para as variáveis categóricas, e o coeficiente de correlação intraclasse, para as variáveis quantitativas, indicaram boa confiabilidade, com resultados variando de 0,60 a 1,00, para o primeiro, e de 0,53 a 0,97 , para o segundo.

\section{Definição das variáveis}

\section{- Variáveis dependentes}

Para avaliação do estado nutricional, as variáveis utilizadas foram o índice de massa corporal (IMC) e razão cintura-estatura. O índice de massa corporal (IMC, $\mathrm{kg} / \mathrm{m}^{2}$ ), obtido pela divisão do peso pela estatura ao quadrado, foi categorizado em quatro grupos $21: \leq 18,5 \mathrm{~kg} / \mathrm{m}^{2}$ (baixo peso), de $\geq 18,5 \mathrm{a}<25,0 \mathrm{~kg} / \mathrm{m}^{2}$ (eutrofia), $\geq 25,0$ $\mathrm{a}<30,0 \mathrm{~kg} / \mathrm{m}^{2}$ (sobrepeso) e $\geq 30,0 \mathrm{~kg} / \mathrm{m}^{2}$ (obesidade). A razão cintura-estatura foi categorizada em $<0,5$ (normal) e $\geq 0,5$ (aumentada) 22,23. Na análise de regressão logística as variáveis IMC e razão cintura-altura foram analisadas como dicotômicas, sendo as variáveis respostas definidas como sobrepeso (IMC $\geq 25,0 \mathrm{~kg} / \mathrm{m}^{2} ; \operatorname{sim} /$ não) e obesidade abdominal (razão cintura-estatura $\geq 0,50$; sim/não).

\section{- Variáveis independentes}

As variáveis independentes foram agrupadas em três blocos: características sociodemográficas, comportamentais e de saúde; todas elas foram autorreferidas, exceto a pressão arterial. Consideraram-se as seguintes características sociodemográficas: sexo (masculino e feminino) faixa etária em anos completos (20-29 anos; 30 39 anos; 40-49 anos; 50-59 anos; 60-69 anos; $\geq$ 70 anos); escolaridade em anos completos de estudo com aprovação (0; 1-3 anos; 4-7 anos; $\geq$ 8 anos); cor da pele autorreferida (preta, parda, branca e outras); renda familiar per capita em

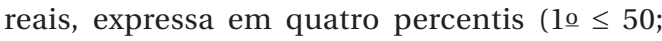
$2 \underline{o}=51-119 ; 3 \underline{o}=120-268 ; 4 \underline{o} \geq 269)$; estado civil (casado/união consensual, nunca foi casado, 
solteiro/separado/viúvo); índice de bens, obtido pela soma total da posse de bens e utensílios domésticos $(\leq 3 ; 4-5 ; \geq 6)$, sendo considerado o relato dos seguintes bens: televisão em cores, fogão a gás, geladeira, freezer, rádio, vídeo/DVD, máquina de lavar roupa, linha de telefone fixo, linha de telefone celular, computador, forno de microondas, carro e motocicleta; situação ocupacional (trabalha, não trabalha); local onde trabalha (zona rural, zona urbana, em ambos os locais); autorreferência como quilombola; e presença de local na vizinhança para comprar frutas e verduras com respostas dicotômicas (sim e não).

As variáveis comportamentais foram: tabagismo (sim e não); tempo assistindo televisão fora do trabalho ( $<2 \mathrm{~h} /$ dia, $\geq 2 \mathrm{~h} /$ dia); nível de atividade física (não realiza atividade física; realiza $>2$ dias/semana por $\geq 30$ minutos); frequência de consumo de frutas e frequência de consumo de verduras/legumes, as quais foram categorizadas em $<5$ dias/semana e $\geq 5$ dias/semana; e comportamento ao comer frango com pele/carne vermelha com gordura, com resposta dicotomizada em sim e não. As características de saúde foram: autoavaliação de saúde (muito boa e boa; regular; ruim e muito ruim); diabetes e depressão autorreferidas (sim e não); hipertensão arterial definida como pressão arterial $\geq 140$ x 90mmHg, obtida por meio da média das duas últimas mensurações de pressão arterial ou uso habitual de medicação anti-hipertensiva.

\section{- Plano analítico}

A amostra incluiu 422 domicílios, totalizando 943 indivíduos. Desse total, 15,5\% ( $\mathrm{n}=146)$ não participaram pelos seguintes motivos: $12,7 \%(n=120)$ não foram encontrados após três visitas e 2,8\% ( $n=26$ ) recusaram. A perda foi maior entre os indivíduos do sexo masculino $(77,4 \% ; \mathrm{n}=89)$ e na faixa etária de $18-34$ anos $(65,2 \% ; n=75)$.

Foram excluídas da presente análise as mulheres grávidas $(1,3 \% ; \mathrm{n}=11)$ e os adolescentes de $10-19$ anos $(5,9 \% ; n=47)$, restando 739 participantes. Mulheres grávidas foram excluídas do estudo por causa da interferência dessa condição nos parâmetros antropométricos.

Inicialmente, foram estimadas as prevalências globais de baixo peso, sobrepeso, obesidade corporal e obesidade abdominal segundo sexo e faixas de idade. Os indivíduos com e sem sobrepeso e com obesidade abdominal foram comparados, considerando-se as variáveis independentes de interesse; a presença de associação estatística foi estimada pelo qui-quadrado de Pearson e nível de 0,05 de significância. As magnitudes das associações entre as variáveis independentes e cada uma das duas variáveis-resposta de interes- se foram determinadas pelo odds ratio (OR), obtido por meio de regressão logística múltipla com IC95\%. O nível de significância estatística de 20\% foi utilizado para a seleção de variáveis candidatas aos modelos multivariáveis, e o de $5 \%$, para o ajuste final. O modelo multivariável final foi construído manualmente, considerando o efeito da entrada de cada variável sobre as demais já retidas no modelo. O teste de Hosmer \& Lemeshow foi usado para avaliação do ajuste dos modelos finais. Para a análise dos dados, empregou-se o software Stata, versão 10.0 (Stata Corp., College Station, Estados Unidos).

O projeto foi aprovado pelos Comitês de Ética em Pesquisa da Faculdade São Francisco de Barreiras (CAAE 0118.0.066.000-10) e pela Universidade Federal de Minas Gerais (CAAE 0118.0.066.203-10). Todos os sujeitos foram informados sobre os objetivos da pesquisa e concordaram em assinar o Termo de Consentimento Livre e Esclarecido.

\section{Resultados}

Entre os 739 adultos elegíveis para o presente estudo, foram excluídos da análise alguns outliers em virtude de erros na mensuração de peso $(2,1 \% ; n=16)$, altura $(3,6 \% ; n=27)$ e circunferência da cintura $(3,9 \% ; n=29)$.

A prevalência global de baixo peso correspondeu a 4\% (IC95\%: 2,5-5,4), sendo ligeiramente maior nos homens $(4,1 \%)$, especialmente nas faixas etárias de 30-49 anos, quando comparados com as mulheres (3,8\%). As prevalências de sobrepeso e obesidade corporal e de razão cintura-estatura aumentada foram maiores entre as mulheres, em todas as faixas de idade, quando comparadas com os homens. As maiores taxas de sobrepeso foram registradas entre as mulheres na faixa de 40-49 anos e entre os homens na faixa de 30-39 anos. Entre os homens, a prevalência global de obesidade corporal foi quase cinco vezes menor que entre as mulheres, tendo sido encontrada apenas nas faixas etárias de 4049 anos e entre aqueles com 60 anos ou mais. A prevalência de razão cintura-estatura aumentada foi maior entre as mulheres do que entre os homens e cresceu conforme a faixa etária nos dois sexos, alcançando quase $80 \%$ nas mulheres idosas (Tabela 1).

As características sociodemográficas estatisticamente associadas ao sobrepeso e também à obesidade abdominal foram sexo, faixa etária, estado civil, e situação ocupacional (Tabela 2). A escolaridade foi associada estatisticamente apenas à obesidade abdominal. Entre os aspectos comportamentais e de saúde, tabagismo, consumo 
Tabela 1

Prevalência do estado nutricional (índice de massa corporal - IMC - e razão cintura-estatura) segundo sexo e faixa etária, em adultos residentes em comunidades quilombolas. Vitória da Conquista, Bahia, Brasil, 2011 ( $n=739$ ).

\begin{tabular}{|c|c|c|c|c|c|c|}
\hline \multirow[t]{2}{*}{ Variável (anos) } & \multicolumn{4}{|c|}{ IMC [\% (IC95\%)] * } & \multicolumn{2}{|c|}{ Razão cintura-estatura [\% (IC95\%)] ** } \\
\hline & Baixo peso & Eutrófico & Sobrepeso & Obesidade & Normal & Aumentada \\
\hline \multicolumn{7}{|l|}{ Feminino } \\
\hline Global & $3,8(1,9-5,7)$ & $43,5(38,6-48,5)$ & $35,8(31,0-40,6)$ & $16,7(13,0-20,4)$ & $34,6(29,8-39,4)$ & $65,3(60,5-70,1)$ \\
\hline $20-29$ & $4,7(0,1-9,2)$ & $60,0(49,5-70,4)$ & $24,7(15,4-33,9)$ & $10,5(3,9-17,1)$ & $60,7(50,1-71,2)$ & $39,2(28,7-49,8)$ \\
\hline $30-39$ & $3,1(0,3-6,6)$ & $41,6(31,7-51,5)$ & $38,5(28,7-48,3)$ & $16,6(9,1-24,7)$ & $38,3(28,4-48,1)$ & $61,7(51,8-71,5)$ \\
\hline $40-49$ & $4,1(0,4-8,7)$ & $34,2(23,2-45,2)$ & $46,5(35,0-58,1)$ & $15,0(6,7-23,3)$ & $27,7(17,3-38,2)$ & $72,2(61,7-82,6)$ \\
\hline $50-59$ & 0,0 & $40,4(26,2-54,6)$ & $40,4(26,2-54,6)$ & $19,1(7,7-30,5)$ & $17,0(6,1-27,9)$ & $82,9(72,0-93,8)$ \\
\hline$\geq 60$ & $5,7(0,8-1,0)$ & $39,0(28,7-49,4)$ & $32,1(22,2-42,0)$ & $22,9(14,0-31,8)$ & $20,2(11,5-28,8)$ & $79,7(71,1-88,4)$ \\
\hline Valor de $\mathrm{p}$ & \multicolumn{4}{|c|}{0,057} & \multicolumn{2}{|c|}{$<0,001$} \\
\hline \multicolumn{7}{|l|}{ Masculino } \\
\hline Global & $4,1(2,0-6,3)$ & $65,8(60,7-70,9)$ & $27,2(22,4-32,0)$ & $2,6(0,9-4,4)$ & $55,4(50,0-60,8)$ & $44,5(39,1-49,9)$ \\
\hline $20-29$ & $3,5(0,1-8,4)$ & $76,7(65,6-87,9)$ & $19,6(9,1-30,1)$ & 0,0 & $87,2(78,3-96,1)$ & $12,7(3,8-21,6)$ \\
\hline $30-39$ & $6,1(0,2-12,0)$ & $58,4(46,3-70,5)$ & $35,3(23,6-47,1)$ & 0,0 & $63,4(51,5-75,4)$ & $36,5(24,5-48,5)$ \\
\hline $40-49$ & $5,4(0,2-10,7)$ & $68,4(57,7-79,2)$ & $21,9(12,3-31,4)$ & $4,1(0,4-8,7)$ & $58,3(46,8-69,8)$ & $41,6(30,1-53,1)$ \\
\hline $50-59$ & $3,5(0,3-8,3)$ & $64,9(52,3-77,4)$ & $31,5(19,3-43,7)$ & 0,0 & $44,6(31,4-57,8)$ & $55,3(42,1-68,5)$ \\
\hline$\geq 60$ & $2,4(0,9-5,7)$ & $62,6(51,1-73,1)$ & $27,7(18,0-37,4)$ & $7,2(1,6-12,8)$ & $32,9(22,6-43,1)$ & $67,0(56,8-77,3)$ \\
\hline Valor de $p$ & \multicolumn{4}{|c|}{0,089} & \multicolumn{2}{|c|}{$<0,001$} \\
\hline \multicolumn{7}{|l|}{ Total } \\
\hline Global & $4,0(2,5-5,4)$ & $53,8(50,2-57,5)$ & $31,8(28,4-35,2)$ & $10,2(8,0-12,4)$ & $44,2(40,6-47,9)$ & $55,7(52,0-59,3)$ \\
\hline $20-29$ & $4,2(0,9-7,6)$ & $66,6(58,8-74,4)$ & $22,7(15,7-29,6)$ & $6,3(2,3-10,4)$ & $71,2(78,3-96,1)$ & $28,7(21,6-38,2)$ \\
\hline $30-39$ & $4,3(1,1-7,5)$ & $48,4(40,6-52,2)$ & $37,2(29,7-44,7)$ & $9,9(5,2-14,5)$ & $48,4(51,5-75,4)$ & $51,5(42,5-58,5)$ \\
\hline $40-49$ & $4,7(1,3-8,2)$ & $51,3(43,2-59,5)$ & $34,2(26,5-41,9)$ & $9,5(4,7-14,3)$ & $43,0(46,8-69,8)$ & $56,9(30,1-58,1)$ \\
\hline $50-59$ & $1,9(0,7-4,5)$ & $53,8(44,2-63,4)$ & $35,5(26,3-44,8)$ & $8,6(3,2-14,0)$ & $32,0(31,4-57,8)$ & $67,9(42,1-68,5)$ \\
\hline$\geq 60$ & $4,1(1,1-7,1)$ & $50,5(43,0-58,1)$ & $30,0(23,0-36,9)$ & $15,2(9,8-20,7)$ & $26,5(22,6-43,1)$ & $73,4(56,8-77,3)$ \\
\hline Valor de $p$ & \multicolumn{4}{|c|}{0,071} & \multicolumn{2}{|c|}{$<0,001$} \\
\hline
\end{tabular}

IC95\%: intervalo de 95\% de confiança.

* IMC: baixo peso $\left(\leq 18,5 \mathrm{~kg} / \mathrm{m}^{2}\right)$, eutrofia $\left(18,5 \geq \mathrm{IMC}<25,0 \mathrm{~kg} / \mathrm{m}^{2}\right)$, sobrepeso $\left(25,0 \geq \mathrm{IMC}<30,0 \mathrm{~kg} / \mathrm{m}^{2}\right)$ e obesidade $\left(\geq 30,0 \mathrm{~kg} / \mathrm{m}^{2}\right)$.

** Razão cintura-estatura: normal $(\leq 0,50)$, aumentada $(\geq 0,50)$.

de carne com gordura e de verduras e legumes em cinco dias ou mais por semana, além de hipertensão arterial, também foram associados ao sobrepeso e à obesidade abdominal. Atividade física, autoavaliação de saúde e relato de diagnóstico de diabetes estiveram associados apenas à obesidade abdominal; tempo assistindo à televisão associou-se ao sobrepeso (Tabela 3 ).

Os fatores associados nas análises uni e multivariável ao sobrepeso e obesidade abdominal são apresentados na Tabela 4. Na análise univariável dos fatores associados ao sobrepeso, a chance de este ocorrer foi mais de duas vezes maior entre as mulheres, em comparação aos homens. Em comparação aos indivíduos com idade entre 20-29 anos, a chance de sobrepeso foi cerca de duas vezes maior em todos os demais grupos etários, sem diferenças estatísticas nas OR das diversas faixas de idade. Indivíduos solteiros e tabagistas apresentaram menores chances de sobrepeso, enquanto comer carne com gordura e ser hipertenso aumentaram esta chance. Todas as associações descritas acima permaneceram estatisticamente significantes na análise multivariável, mas as associações com consumo de carne com gordura e o relato de hipertensão foram limítrofes ( $\mathrm{p}=0,58 \mathrm{e}$ $\mathrm{p}=0,53$, respectivamente).

No que diz respeito à obesidade abdominal, na análise univariável, os indivíduos do sexo feminino, com idade mais avançada ( $\geq 60$ anos), baixa escolaridade (1-4 anos de estudo), que não trabalhavam, consumiam carne com gordura e sofriam de hipertensão arterial apresentaram maiores chances de ter obesidade abdominal. Já entre os indivíduos solteiros e tabagistas, a 
Tabela 2

Distribuição amostral e prevalência de sobrepeso e obesidade abdominal segundo variáveis sociodemográficas e econômicas, entre adultos residentes de comunidades quilombolas. Vitória da Conquista, Bahia, Brasil, 2011.

\begin{tabular}{|c|c|c|c|}
\hline Variáveis & $\begin{array}{c}\text { Distribuição } \\
\text { amostral [n (\%)] }\end{array}$ & $\begin{array}{l}\text { Sobrepeso } \\
\text { (n= 722)* } \\
\%(\text { IC95\%) }\end{array}$ & $\begin{array}{l}\text { Obesidade abdominal } \\
\qquad \begin{array}{c}(n=709) * * \\
\%(I C 95 \%)\end{array}\end{array}$ \\
\hline Sexo & & $p<0,001$ & $p<0,001$ \\
\hline Masculino & $343(46,4)$ & $29,9(25,0-34,8)$ & $44,5(39,1-49,9)$ \\
\hline Feminino & $396(53,5)$ & $52,5(47,5-57,5)$ & $65,3(60,5-70,1)$ \\
\hline Faixa etária (anos) & & $p=0,013$ & $p<0,001$ \\
\hline $20-29$ & $144(19,4)$ & $29,0(21,5-36,6)$ & $28,7(21,2-36,3)$ \\
\hline $30-39$ & $164(22,1)$ & $47,2(39,4-54,9)$ & $51,5(43,7-59,4)$ \\
\hline $40-49$ & $150(20,3)$ & $43,8(35,7-51,9)$ & $56,9(48,8-65,0)$ \\
\hline $50-59$ & $104(14,0)$ & $44,2(34,6-53,8)$ & $67,9(58,8-77,0)$ \\
\hline$\geq 60$ & $177(23,9)$ & $45,2(37,5-32,8)$ & $73,4(66,7-80,2)$ \\
\hline Escolaridade (anos) & & $p=0,262$ & $p<0,001$ \\
\hline 0 & $271(36,6)$ & $39,6(33,6-45,6)$ & $60,7(54,7-66,7)$ \\
\hline $1-3$ & $210(28,4)$ & $46,1(39,4-52,9)$ & $61,1(54,4-67,8)$ \\
\hline $4-7$ & $185(25,0)$ & $43,8(36,6-51,1)$ & $50,0(42,6-57,3)$ \\
\hline$\geq 8$ & $68(9,6)$ & $34,3(22,8-45,8)$ & $32,3(20,8-43,7)$ \\
\hline Cor da pele & & $p=0,743$ & $p=0,324$ \\
\hline Preta & $287(39,3)$ & $42,7(36,9-48,5)$ & $53,2(47,3-59,1)$ \\
\hline Parda & $325(44,5)$ & $42,0(36,6-47,5)$ & $58,9(53,4-64,4)$ \\
\hline Branco & $97(13,3)$ & $42,1(32,1-52,1)$ & $54,8(44,6-65,0)$ \\
\hline Outras & $20(2,7)$ & $30,0(9,3-50,6)$ & $42,1(19,2-64,9)$ \\
\hline Quilombola & & $p=0,763$ & $p=0,277$ \\
\hline Não & $111(15,2)$ & $38,7(29,6-49,8)$ & $41,4(32,2-50,6)$ \\
\hline Sim & $616(84,7)$ & $40,2(36,3-44,1)$ & $36,0(32,2-39,8)$ \\
\hline Renda (R\$) & & $p=0,267$ & $p=0,450$ \\
\hline$\leq 50,00$ & $184(26,6)$ & $38,6(31,4-45,8)$ & $53,4(46,0-60,8)$ \\
\hline $51,00-119,00$ & $144(20,8)$ & $43,9(35,7-52,2)$ & $58,9(50,7-67,2)$ \\
\hline $120,00-268,00$ & $180(26,0)$ & $46,8(35,5-54,2)$ & $55,2(51,8-66,5)$ \\
\hline$\geq 269,00$ & $182(26,3)$ & $37,9(30,8-45,1)$ & $52,3(44,8-59,5)$ \\
\hline Índice de bens & & $p=0,342$ & $p=0,133$ \\
\hline$\leq 3$ & $236(31,9)$ & $38,8(32,4-45,2)$ & $50,6(44,0-57,2)$ \\
\hline $4-5$ & $293(39,6)$ & $45,1(39,3-50,9)$ & $56,1(50,3-61,9)$ \\
\hline$\geq 6$ & $210(28,4)$ & $41,1(34,4-47,8)$ & $60,2(55,5-67,0)$ \\
\hline Estado civil & & $p=0,002$ & $p<0,001$ \\
\hline Casado/União consensual & $473(64,0)$ & $46,4(41,9-50,9)$ & $61,8(57,4-66,3)$ \\
\hline Nunca foi casado & $148(20,0)$ & $29,8(22,3-37,3)$ & $30,2(22,5-37,8)$ \\
\hline Separado/Divorciado/Viúvo & $118(15,9)$ & $39,8(30,7-48,9)$ & $62,1(53,0-71,2)$ \\
\hline Situação ocupacional & & $p<0,001$ & $p<0,001$ \\
\hline Trabalha & $365(49,3)$ & $35,0(30,1-39,9)$ & $45,5(40,3-50,0)$ \\
\hline Não trabalha & $374(50,6)$ & $49,3(44,0-54,5)$ & $66,1(61,1-71,0)$ \\
\hline Local onde trabalha & & $p=0,370$ & $p=0,244$ \\
\hline Zona rural & $501(76,4)$ & $39,3(35,0-43,7)$ & $55,5(51,1-60,0)$ \\
\hline Zona urbana & $99(15,1)$ & $41,6(31,7-51,5)$ & $50,5(40,3-60,7)$ \\
\hline Ambos os locais & $55(8,4)$ & $49,0(35,7-62,4)$ & $64,8(51,9-77,6)$ \\
\hline Local para comprar frutas e verduras & & $p=0,492$ & $p=0,535$ \\
\hline Não & $599(81,0)$ & $42,6(38,6-46,6)$ & $56,2(52,1-60,2)$ \\
\hline Sim & $140(18,9)$ & $39,4(31,1-47,6)$ & $53,2(44,8-61,6)$ \\
\hline
\end{tabular}

IC95\%: intervalo de $95 \%$ de confiança.

* Sobrepeso (índice de massa corporal $\geq 25,0 \mathrm{~kg} / \mathrm{m}^{2}$ );

** Obesidade abdominal (razão cintura-estatura $\geq 0,50$ ).

Nota: a soma do número total varia devido a perdas de informações. 
Distribuição amostral e prevalência de sobrepeso e obesidade abdominal segundo variáveis comportamentais e de saúde, entre adultos residentes de comunidades quilombolas. Vitória da Conquista, Bahia, Brasil, 2011.

\begin{tabular}{|c|c|c|c|}
\hline Variáveis & $\begin{array}{l}\text { Distribuição amostral } \\
\text { [n (\%)] }\end{array}$ & $\begin{array}{l}\text { Sobrepeso } \\
(n=722) \text { * } \\
\%(I C 95 \%)\end{array}$ & $\begin{array}{l}\text { Obesidade abdominal } \\
\qquad \begin{array}{c}(n=709) * \star \\
\%(I C 95 \%)\end{array}\end{array}$ \\
\hline Tabagismo & & $p<0,001$ & $p<0,001$ \\
\hline Não & $585(79,1)$ & $46,5(38,5-46,6)$ & $59,7(55,6-63,8)$ \\
\hline Sim & $154(20,8)$ & $25,3(18,4-32,2)$ & $40,6(32,7-48,5)$ \\
\hline Atividade física & & $p=0,645$ & $p=0,013$ \\
\hline Não realiza atividade física & $569(77,3)$ & $41,6(37,5-45,7)$ & $58,2(54,1-62,4)$ \\
\hline $\begin{array}{l}>2 \text { dias/semana por } \geq 30 \\
\text { minutos }\end{array}$ & $167(22,6)$ & $43,6(36,0-51,2)$ & $47,2(39,5-54,9)$ \\
\hline Tempo de televisão (horas/dia) & & $p=0,047$ & $p=0,110$ \\
\hline$<2$ & $372(50,8)$ & $42,3(38,3-46,3)$ & $52,9(47,7-59,2)$ \\
\hline$\geq 2$ & $359(49,1)$ & $41,7(32,6-49,9)$ & $58,9(53,8-64,1)$ \\
\hline Consumo de frutas (dias/semanas) & & $p=0,827$ & $p=0,384$ \\
\hline$<5$ & $603(81,9)$ & $39,62(35,6-43,5)$ & $55,0(51,0-59,1)$ \\
\hline$\geq 5$ & $133(18,0)$ & $34,9(26,5-43,2)$ & $59,3(50,6-68,0)$ \\
\hline $\begin{array}{l}\text { Consumo de verduras/legumes } \\
\text { (dias/semanas) }\end{array}$ & & $p=0,058$ & $p=0,051$ \\
\hline$<5$ & $636(86,4)$ & $40,7(36,9-44,6)$ & $54,4(50,4-58,3)$ \\
\hline$\geq 5$ & $100(13,5)$ & $51,0(40,9-61,1)$ & $65,2(55,4-75,0)$ \\
\hline $\begin{array}{l}\text { Consumo de frango com pele/ } \\
\text { carne vermelha com gordura }\end{array}$ & & $p=0,003$ & $p=0,001$ \\
\hline Não & $417(58,5)$ & $37,9(33,1-42,6)$ & $51,1(46,2-56,0)$ \\
\hline Sim & $295(41,4)$ & $49,3(43,4-55,1)$ & $63,8(58,1-69,4)$ \\
\hline Autoavaliação da saúde & & $p=0,257$ & $p=0,019$ \\
\hline Muito boa/Boa & $317(43,0)$ & $38,9(33,5-44,3)$ & $49,8(44,1-55,4)$ \\
\hline Regular & $319(43,3)$ & $45,4(39,9-51,0)$ & $60,9(55,5-66,4)$ \\
\hline Ruim /Muito ruim & $100(13,5)$ & $41,6(31,7-51,5)$ & $58,0(47,9-68,1)$ \\
\hline Hipertensão arterial & & $p=0,003$ & $p \leq 0,001$ \\
\hline Não & $378(51,6)$ & $36,9(30,1-39,8)$ & $45,0(39,9-50,1)$ \\
\hline Sim & $354(48,6)$ & $47,8(42,5-53,1)$ & $67,4(62,4-72,4)$ \\
\hline Diabetes mellitus & & $p=0,101$ & $p=0,024$ \\
\hline Não & $457(91,4)$ & $45,2(40,5-49,8)$ & $60,1(55,5-64,7)$ \\
\hline $\operatorname{Sim}$ & $43(8,6)$ & $58,5(43,2-73,8)$ & $78,0(65,1-90,9)$ \\
\hline Depressão & & $p=0,492$ & $p=0,794$ \\
\hline Não & $721(97,5)$ & $41,90(38,2-45,5)$ & $55,6(51,9-59,3)$ \\
\hline Sim & $18(2,4)$ & $50,0(26,1-73,8)$ & $58,8(34,6-82,9)$ \\
\hline
\end{tabular}

IC95\%: intervalo de 95\% de confiança.

* Sobrepeso (índice de massa corporal > 25,0kg/m2);

** Obesidade abdominal (razão cintura-estatura $>0,50$ ).

Nota: a soma do número total varia devido a perdas de informações.

chance de obesidade abdominal foi menor. Todas as associações descritas permaneceram estatisticamente significantes na análise multivariável (Tabela 4 ).
Os ajustes dos modelos pelo teste de Hosmer $\&$ Lemeshow foram adequados com valores de $\mathrm{p}=0,81$ para o sobrepeso e $\mathrm{p}=0,56$ para a obesidade abdominal. 
Odds ratio (OR) bruta e ajustada do sobrepeso e obesidade abdominal em adultos residentes em comunidades quilombolas, segundo variáveis sociodemográficas, comportamentais e de saúde. Vitória da Conquista, Bahia, Brasil, 2011.

\begin{tabular}{|c|c|c|c|c|}
\hline \multirow[t]{2}{*}{ Variáveis } & \multicolumn{2}{|c|}{ Sobrepeso * } & \multicolumn{2}{|c|}{ Obesidade abdominal ** } \\
\hline & $\begin{array}{l}\text { OR bruto } \\
\text { (IC95\%) }\end{array}$ & 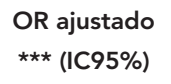 & $\begin{array}{l}\text { OR bruto } \\
\text { (IC95\%) }\end{array}$ & $\begin{array}{l}\text { OR ajustado } \\
\star \star \star ~(I C 95 \%)\end{array}$ \\
\hline \multicolumn{5}{|l|}{ Sexo } \\
\hline Masculino & 1,0 & 1,0 & 1,0 & 1,0 \\
\hline Feminino & $2,5(1,9-3,5)$ & $2,2(1,6-3,1)$ & $2,3(1,7-3,1)$ & $2,0(1,3-3,1)$ \\
\hline \multicolumn{5}{|l|}{ Faixa etária (anos) } \\
\hline $20-29$ & 1,0 & 1,0 & 1,0 & 1,0 \\
\hline $30-39$ & $2,1(1,3-3,5)$ & $2,2(1,3-3,7)$ & $2,6(1,6-4,2)$ & $2,9(1,6-5,2)$ \\
\hline $40-49$ & $1,9(1,1-3,1)$ & $1,9(0,9-3,4)$ & $3,2(1,9-5,3)$ & $4,2(2,2-7,8)$ \\
\hline $50-59$ & $1,9(1,1-3,2)$ & $1,8(1,0-3,4)$ & $5,2(3,0-9,1)$ & $5,9(2,9-12,1)$ \\
\hline$\geq 60$ & $2,0(1,2-3,2)$ & $1,7(0,9-3,1)$ & $6,8(4,1-11,3)$ & $7,5(3,6-15,5)$ \\
\hline \multicolumn{5}{|l|}{ Escolaridade (anos de estudo) } \\
\hline 0 & & & 1,0 & 1,0 \\
\hline $1-4$ & & & $1,0(0,6-1,4)$ & $1,6(1,0-2,5)$ \\
\hline $4-7$ & & & $0,6(0,4-0,9)$ & $1,4(0,8-2,3)$ \\
\hline$\geq 8$ & & & $0,3(0,1-0,5)$ & $1,4(0,6-3,1)$ \\
\hline \multicolumn{5}{|l|}{ Estado civil } \\
\hline Casado/União consensual & 1,0 & 1,0 & 1,0 & 1,0 \\
\hline Nunca foi casado & $0,4(0,3-0,7)$ & $0,6(0,4-0,8)$ & $0,2(0,1-0,4)$ & $0,3(0,2-0,6)$ \\
\hline Separado/Divorciado/Viúvo & $0,7(0,5-1,1)$ & $0,6(0,3-1,0)$ & $1,0(0,6-1,5)$ & $0,7(0,4-1,3)$ \\
\hline \multicolumn{5}{|l|}{ Situação ocupacional } \\
\hline Trabalha & & & 1,0 & 1,0 \\
\hline Não trabalha & & & $2,2(1,6-3,0)$ & $1,5(1,0-2,4)$ \\
\hline \multicolumn{5}{|l|}{ Tabagismo } \\
\hline Não & 1,0 & 1,0 & 1,0 & 1,0 \\
\hline Sim & $0,3(0,2-0,5)$ & $0,4(0,3-0,7)$ & $0,4(0,3-0,6)$ & $0,5(0,3-0,8)$ \\
\hline \multicolumn{5}{|c|}{$\begin{array}{l}\text { Consumo de frango com pele/carne vermelha } \\
\text { com gordura }\end{array}$} \\
\hline Não & 1,0 & 1,0 & 1,0 & 1,0 \\
\hline Sim & $1,5(1,1-2,1)$ & $1,3(0,9-1,9)$ & $1,6(1,2-2,3)$ & $1,4(1,0-2,1)$ \\
\hline \multicolumn{5}{|l|}{ Hipertensão arterial } \\
\hline Não & 1,0 & 1,0 & 1,0 & 1,0 \\
\hline Sim & $1,5(1,1-2,1)$ & $1,4(0,9-2,0)$ & $2,5(1,8-3,4)$ & $1,5(1,0-2,2)$ \\
\hline \multicolumn{5}{|c|}{ IC95\%: intervalo de 95\% de confiança. } \\
\hline \multicolumn{5}{|c|}{ * Sobrepeso (índice de massa corporal > 25,0kg/m²); } \\
\hline \multicolumn{5}{|c|}{ 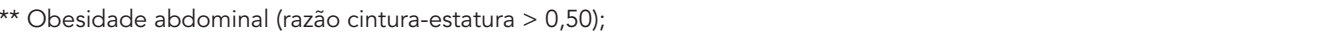 } \\
\hline ** OR ajustado por todas as variá & & & & \\
\hline
\end{tabular}

\section{Discussão}

Os achados do presente estudo indicam que o sobrepeso e a obesidade abdominal constituem importantes problemas de saúde nas comunidades quilombolas e, mais especificamente, entre as mulheres. Esses resultados estão em consonância com o crescimento da obesidade corporal e central em áreas mais pobres do Brasil 24,25,26, especialmente entre determinados grupos vulneráveis $4,5,27$.

As maiores prevalências de sobrepeso e de obesidade abdominal identificadas entre as mulheres quilombolas são consistentes com achados de outros trabalhos desenvolvidos com populações de matriz africana 28,29,30,31 e com outras populações em geral 27,32,33,34,35. A prevalência de sobrepeso (IMC $\geq 25,0 \mathrm{~kg} / \mathrm{m}^{2}$ ) foi maior 
entre as mulheres quilombolas no presente trabalho (52,5\%) em comparação à descrita para mulheres no país $(48 \%)$ e no Nordeste $(43,5 \%)$ pela POF de 2008-2009 8. Com relação à obesidade, a prevalência entre as mulheres quilombolas (16,7\%) está bem próxima da registrada para esse grupo na POF (16,9\%) e no Nordeste $(15,2 \%)$, e um pouco superior à descrita para mulheres na zona rural desta mesma região (13,8\%). No sexo masculino, observa-se o contrário. A prevalência de sobrepeso e, especialmente, a de obesidade foram menores entre os homens quilombolas em comparação ao encontrado pela POF neste segmento populacional no país $(27,2 \%$ s. $50,1 \%$ e $2,6 \%$ vs. $12,5 \%$ ) e na zona rural do Nordeste (5,7\%). Nossos resultados mostram ainda que, nesta pesquisa, a prevalência de baixo peso entre os homens $(4,1 \%)$ é superior à descrita na $\mathrm{POF}$ $(1,8 \%)$ para o mesmo grupo.

Estudo com remanescentes de quilombos do Vale do Ribeira, São Paulo 6, apontou o trabalho como outra possível explicação para as diferenças de gênero encontradas. Os homens trabalham mais na agricultura de subsistência, desenvolvendo atividades físicas laborais mais intensas, com pouco ou nenhum insumo tecnológico, o que resulta em maior dispêndio de energia, enquanto as mulheres declararam ocupar-se mais de afazeres domésticos.

A associação entre sexo feminino e obesidade abdominal pode refletir, em parte, o acúmulo de gordura na região do abdômen devido à ocorrência de gravidez, além de modificações metabólicas e hormonais ao longo da vida 36 . Estudo desenvolvido com mulheres afro-americanas mostrou que a chance de obesidade entre elas foi duas vezes maior do que a observada entre outras mulheres de origem pobre, refletindo possível desvantagem ao longo da vida 37 .

Assim como em outros estudos de base populacional 32,35 , as prevalências globais de obesidade corporal e obesidade central aumentaram com o envelhecimento da população quilombola. Com o avançar da idade, há uma tendência de perda de massa magra, aumento da proporção de gordura corpórea, diminuição da estatura, relaxamento da musculatura abdominal, cifose, dentre outros 38 , mudanças que afetam o IMC e a razão cintura-estatura. Estudos demonstram que, com o avançar da idade, as mulheres tendem a acumular mais gordura subcutânea do que os homens 39 .

A elevação da prevalência da obesidade central com o avanço da idade é consistente com outros trabalhos 35 . O fato de a razão cintura-estatura levar em conta a altura corporal corrige, de certo modo, as diferenças na circunferência da cintura em decorrência de variações na esta- tura. Por isso, entre as medidas de adiposidade central, a utilização da razão cintura-estatura parece mais adequada 22,23 , uma vez que a fixação de pontos de corte para a circunferência da cintura sem considerar a estatura pode subestimar a gordura abdominal em indivíduos baixos e superestimar em indivíduos mais altos 40 . Ademais, vale destacar que a menor estatura, inclusive em adultos, pode ser um marcador de desnutrição na infância e tem sido associada com a obesidade em alguns estudos 24,41 . Por outro lado, a elevação da prevalência de obesidade abdominal nos idosos também é afetada pela redução da estatura com o envelhecimento, já que a altura é denominador dessa medida. A redução da estatura pode se dever a alterações ósseas, como achatamento das vértebras, redução dos discos intervertebrais, cifose dorsal, escoliose, arqueamento dos membros inferiores e/ ou achatamento do arco plantar 42,43 .

A prevalência de sobrepeso foi mais alta na faixa etária de 30-39 anos, para homens, e de 4049 anos, para as mulheres, faixas mais precoces do que as observadas na POF em 2008-2009 8 . No referido inquérito, as prevalências de sobrepeso e obesidade aumentaram com a idade até as faixas etárias de 45-54 anos, em homens, e de 55-64 anos, em mulheres, declinando nas idades subsequentes ${ }^{8}$. A queda do sobrepeso entre os mais velhos pode expressar menor sobrevivência destes, já que o sobrepeso e obesidade contribuem para aumentar a mortalidade nesta faixa etária 39.

A permanência da variável escolaridade na análise ajustada confirmou seu papel como preditor independente da adiposidade central: quilombolas com menor escolaridade apresentaram mais chance de obesidade abdominal. Nas últimas décadas, a prevalência de obesidade tem crescido entre os indivíduos com menor escolaridade mais do que entre os demais: no período 1975-1989, a prevalência de obesidade não diferia por escolaridade; no período 1989-1997, a obesidade aparece mais alta entre os indivíduos com menor escolaridade 44. Dados do VIGITEL de 2006-2009 45 também mostram maior prevalência de sobrepeso e obesidade entre mulheres com menor escolaridade, mas o mesmo não foi identificado entre os homens. Além de ser um importante indicador de condição socioeconômica geral, indivíduos com menor escolaridade geralmente têm menos acesso a informações e orientações sobre como preservar a saúde e prevenir doenças 46 .

No presente estudo, as prevalências de sobrepeso e de obesidade abdominal foram menores entre os que trabalhavam. Indivíduos que estão trabalhando tendem a ter maiores níveis 
de atividade física no trabalho, o que pode levar a maior gasto energético e explicar os resultados encontrados. Por outro lado, o tipo de atividade laboral parece exercer influência também, conforme estudo em São Paulo 6, o qual apontou que a vinculação de mulheres quilombolas com o trabalho doméstico diminuiu o seu tempo livre para a realização de atividade física, contribuindo para uma maior inatividade física fora do trabalho.

A menor prevalência de sobrepeso entre os solteiros e os separados/viúvos também foi encontrada em trabalho realizado nas capitais brasileiras e no Distrito Federal 47 . É possível que indivíduos casados ou que vivem com companheiro/a realizem menos atividade física geral, seja por disporem de pouco tempo livre, seja por gastarem mais tempo cuidando da família e da casa.

O consumo de carne com gordura se manteve associado com os desfechos analisados. Apesar de o consumo de gordura saturada, como o toucinho, ter sido apontado como hábito bastante difundido entre os africanos escravizados 11, não se pode atribuir a associação entre consumo de carne com gordura e o sobrepeso e obesidade abdominal apenas a uma herança alimentar incorporada. Lignani et al. 48 identificaram que indivíduos de baixa renda e escolaridade que sejam beneficiários de programas de transferência de renda no Brasil apresentaram aumento no consumo de alimentos não saudáveis. Outro elemento importante é que, nas últimas três décadas, os padrões de consumo alimentar por regiões metropolitanas do Brasil têm apontado tendências desfavoráveis: o consumo de alimentos básicos e tradicionais tem declinado, enquanto alimentos industrializados e gorduras em geral e saturadas passaram a ser mais ingeridos 49 .

Não há consenso sobre a relação entre hábito de fumar, de um lado, e sobrepeso 50 e obesidade abdominal 51, de outro. No presente trabalho, o tabagismo apresentou associação protetora para sobrepeso e obesidade abdominal, resultado que está em consonância com os estudos anteriormente mencionados. Uma das explicações para tal associação seria o fato de o tabaco competir com os sítios de recompensa cerebral dos alimentos, reduzindo o apetite; efeito semelhante apresenta a nicotina, que aumenta a oxidação de lipídeos.

A associação entre hipertensão e obesidade é bem estabelecida 47,52 . Nesta investigação, a hipertensão foi associada com a obesidade abdominal e apresentou uma significância limítrofe para o sobrepeso.

Um aspecto que se destaca no presente estudo é a autodescrição da população analisada com relação à cor da pele/raça: apesar do predomínio de pretos e do grande contingente de pardos, a presença de brancos sugere a existência de intercâmbios migratórios, de cruzamentos inter-raciais e a redução do isolamento destas comunidades quilombolas. Tanto o critério de autoclassificação, utilizado nesta pesquisa, como o de heteroclassificação podem "branquear" ou "escurecer" indivíduos, dependendo do contexto, da região do país e da posição social 53, influenciando, assim, os resultados encontrados. Estudos em Pelotas, Rio Grande do Sul 54, e no Rio de Janeiro 53 indicam que o fenômeno do branqueamento tende a decorrer de melhores condições socioeconômicas.

Algumas limitações potenciais necessitam ser consideradas na interpretação dos resultados deste estudo. A primeira relaciona-se com a ausência de aferição da confiabilidade das medidas antropométricas utilizadas para construir o IMC e a razão cintura-estatura. Essa falta foi minimizada pela padronização de procedimentos e por medidas de garantia e de controle de qualidade. Dessa forma, possíveis erros de medida certamente foram não diferenciais, tornando as associações encontradas conservadoras. Outra limitação é inerente aos estudos transversais, uma vez que estes não permitem estabelecer relação temporal entre exposição e desfecho; no entanto, essa verificação não foi objetivo deste estudo. Por fim, as maiores taxas de não resposta entre homens e entre os mais jovens podem ter comprometido a aferição das diferenças de prevalências de sobrepeso e obesidade abdominal entre os sexos. Em adição, tais perdas podem estar associadas, também, à distribuição de outros fatores, como trabalho e escolaridade, que variam com o gênero e a idade.

\section{Considerações finais}

Os resultados mostraram que as alterações nutricionais de maior relevância entre os adultos quilombolas foram o sobrepeso e o acúmulo de gordura abdominal, acentuadamente entre as mulheres. As associações entre sobrepeso e obesidade abdominal, e fatores sociodemográficos, comportamentais e de saúde investigados, refletem o emaranhado complexo de variáveis que fazem parte da história natural desses dois importantes problemas de saúde nessa população rural, predominantemente de cor/raça preta e homogeneamente pobre. $\mathrm{O}$ uso de mais de um indicador antropométrico ofereceu informação adicional para mapear o risco de doenças crônicas não transmissíveis associadas ao sobrepeso e à adiposidade central. A formação e perpetua- 
ção das comunidades quilombolas existentes, a precariedade das condições socioeconômicas e o perfil nutricional mostrados neste estudo reforçam a necessidade de políticas públicas voltadas à promoção de dietas e modos de vida saudáveis que respeitem e valorizem as especificidades culturais e sociais dessas comunidades.

\section{Resumen}

Este estudio caracteriza el estado nutricional, las estimaciones de la prevalencia del sobrepeso y la obesidad abdominal e investiga los factores asociados. Dos etapas muestra aleatoria de 739 adultos de $>20$ años, que viven en comunidades de los quilombos en Vitória da Conquista, Bahía, Brasil en 2011. La prevalencia del sobrepeso y la obesidad corporal eran el 31,8\% y el 10,2\% respectivamente y el aumento de la cintura a la estatura $(>0,50) 55,7 \%$. El sexo femenino, el consumo de pollo o carne con la grasa y la hipertensión aumentan las probabilidades para los dos indicadores, mientras que el tabaquismo y estado civil soltero disminuyó esta oportunidad. El sobrepeso fue mayor en el grupo de edad de 30-39 años, y la obesidad abdominal fue más frecuente en edades avanzadas. A poca educación y sin trabajo aumentan las probabilidades de obesidad abdominal. La prevalencia de estos eventos son altos en una población de alta vulnerabilidad social, lo que indica la necesidad de adoptar medidas específicas para prevenir y controlar la obesidad.

Sobrepeso; Obesidad Abdominal; Estatura; Grupo de Ascendencia Continental Africana

\section{Colaboradores}

D. A. Soares e S. M. Barreto contribuíram desde a concepção do projeto até a análise, interpretação dos dados, redação do artigo, revisão crítica e aprovação final da versão a ser publicada.

\section{Agradecimentos}

Este trabalho foi financiado pela FAPESB conforme edital 20/2010. Agradecemos a todos os participantes do Projeto COMQUISTA por colaborarem na elaboração, planejamento e supervisão da coleta de dados do projeto. Agradecemos à CAPES, pelo financiamento do Doutorado Interinstitucional (DINTER), por meio do Edital 05/2009, Ação Novas Fronteiras. 


\section{Referências}

1. Schmidt MI, Duncan BB, Silva GA, Menezes AM Monteiro CA, Barreto SM, et al. Chronic non-communicable diseases in Brazil: burder and current challenges. Lancet 2011; 377:1949-61.

2. Candib LM. Obesity and diabetes in vulnerable populations: reflection on proximal and distal causes. Ann Fam Med 2007; 5:547-56.

3. Batista Filho M, Souza AI, Miglioli TC, Santos MC. Anemia e obesidade: um paradoxo da transição nutricional brasileira. Cad Saúde Pública 2008; 24 Suppl 2:S247-57.

4. Salvo VLMA, Rodrigues D, Baruzzi RG, Gimeno SGA. Perfil metabólico e antropométrico dos Suyá. Parque indígena do Xingu, Brasil Central. Rev Bras Epidemiol 2009; 12:458-68.

5. Gimeno SGA, Rodrigues D, Pagliaro H, Cano EN, Lima EES, Baruzzi RG. Perfil metabólico e antropométrico de índios Aruák: Mehináku, Waurá e Yawalapití, Alto Xingu, Brasil Central, 2000/2002. Cad Saúde Pública 2007; 23:1946-54.

6. Volochko A, Batista LE. Saúde nos quilombos. São Paulo: Instituto de Saúde, Secretaria Estadual da Saúde de São Paulo; 2009. (Temas em Saúde Coletiva, 9).

7. Silva JAN. Condições sanitárias e de saúde em Caiana dos Crioulos, uma comunidade quilombola do estado da Paraíba. Saúde Soc 2007; 16: 111-24.

8. Instituto Brasileiro de Geografia e Estatística. Pesquisa de Orçamentos Familiares - POF 2008/2009. Antropometria e estado nutricional de crianças, adolescentes e adultos no Brasil. Rio de Janeiro: Instituto Brasileiro de Geografia e Estatística; 2010

9. Instituto de Pesquisa Econômica Aplicada. Quilombos das Américas: articulação de comunidades afrorrurais: documento síntese. Brasília: Instituto de Pesquisa Econômica Aplicada; 2012.

10. Silva DO, Guerreiro AFH, Guerrero CH, Toledo LM. A rede de causalidade da insegurança alimentar e nutricional de comunidades quilombolas com a construção da rodovia BR-163, Pará, Brasil. Rev Nutr 2008; 21 Suppl:S83-97.

11. Pires CGS, Mussi FC. Crenças em saúde sobre dieta: uma perspectiva de pessoas negras hipertensas. Rev Esc Enferm USP 2012; 46:580-9.

12. Loner BA, Gill LA, Scheer MI. Enfermidade e morte: os escravos na cidade de Pelotas, 1870-1880. Hist Ciênc Saúde-Manguinhos 2012; 19 Suppl 1:S133-52.

13. Moura PG, Batista LRV, Moreira EAM. População indígena: uma reflexão sobre a influência da civilização urbana no estado nutricional e na saúde bucal. Rev Nutr 2010; 23:459-65.

14. Voster HH. The emergence of cardiovascular disease during urbanization of Africans. Public Health Nutr 2002; 5:239-43.

15. Guerrero AFH. Situação nutricional de populações remanescentes de quilombos do município de Santarém - Pará, Brasil [Tese de Doutorado]. Rio de Janeiro: Escola Nacional de Saúde Pública Sergio Arouca, Fundação Oswaldo Cruz; 2010.
16. Leite IB. O projeto político quilombola: desafios, conquistas e impasses atuais. Revista Estudos Feministas 2008; 16:965-77.

17. Fundação Palmares. Comunidades quilombolas de Vitória da Conquista, Bahia. 2011 http://www. palmares.gov.br/quilombola/?estado=BA (acessado em 12/Jan/2011)

18. Verger P. Fluxo e refluxo do tráfico de escravos entre o Golfo do Benin e a Bahia de Todos os Santos: dos séculos XVII a XIX. Salvador: Corrupio; 2002.

19. Jelliffe DB. The assessment of nutritional status of the community. Geneva: World Health Organization; 1966.

20. Sociedade Brasileira de Cardiologia; Sociedade Brasileira de Hipertensão; Sociedade Brasileira de Nefrologia. VI diretrizes brasileiras de hipertensão. Arq Bras Cardiol 2010; 95(1 Suppl 1):1-51.

21. World Health Organization. Obesity: preventing and managing the global epidemic. Report of a WHO consultation on obesity. Geneva: World Health Organization; 1998.

22. Ashwell M, Hsieh SD. Six reasons why the waist-toheight ratio is a rapid and effective global indicator for health risks of obesity and how its use could simplify the international public health message on obesity. Int J Food Sci Nutr 2005; 56:303-7.

23. Ashwell M, Gunn P, Gibson S. Waist-to-height ratio is a better screening tool than waist circumference and BMI for adult cardiometabolic risk factors: systematic review and meta-analysis. Obes Rev 2012; 13:275-86.

24. Alves JG, Falcão RW, Pinto RA, Correia JB. Obesity patterns among women in a slum area in Brazil. J Health Popul Nutr 2011; 29:286-9

25. Monteiro CA, Conde WL, Popkin BM. Incomespecifc trends in Obesity in Brazil: 1975-2003. Am J Public Health 2007; 97:1808-12.

26. Rocha AKS, Bós AJG, Huttner E, Machado DC. Prevalência da síndrome metabólica em indígenas com mais de 40 anos no Rio Grande do Sul, Brasil. Rev Panam Salud Pública 2011; 29:41-5.

27. Lourenço AE, Santos RV, Orellana JD, Coimbra Jr. CEA. Nutrition transition in Amazonia: obesity and socioeconomic change in the Suruí Indians from Brazil. Am J Hum Biol 2008; 20:564-71.

28. Kruger A, Wissing MP, Towers GW, Doak CM. Sex differences independent of other psycho-sociodemographic factors as a predictor of body mass index in black South African Adults. J Health Popul Nutr 2012; 30:56-65.

29. Motala AA, Esterhuizen T, Pirie FJ, Omar MAK. The prevalence of metabolic syndrome and determination of the optimal waist circumference cutoff points in a rural South African Community. Diabetes Care 2011; 34:1032-7.

30. Puoane T, Steyn K, Bradshaw D, Laubscher R, Fourie J, Lambert V, et al. Obesity in South Africa: the South African demographic and health survey. Obes Res 2002; 10:1038-48. 
31. Cappuccio FP, Kerry SM, Adeyemo A, Luke A, Amoah AGB, Bovet P, et al. Body size and blood pressure: an analysis of Africans and the African diaspora. Epidemiology 2008; 19:38-46.

32. Oliveira LPM, Assis AMO, Silva MCM, Santana MLP, Santos NS, Pinheiro SMC, et al. Fatores associados a excesso de peso e concentração de gordura abdominal em adultos na cidade de Salvador, Bahia, Brasil. Cad Saúde Pública 2009; 25:570-82.

33. Olinto MTA, Nácul LC, Dias-da-Costa JS, Gigante DP, Menezes AMB, Macedo S. Níveis de intervenção para a obesidade abdominal: prevalência e fatores associados. Cad Saúde Pública 2006; 22:1207-15.

34. Gigante DP, Dias-da-Costa JS, Olinto MTA, Menezes AMB, Macedo S. Obesidade da população adulta de Pelotas, Rio Grande do Sul, Brasil e associação com nível sócio-econômico. Cad Saúde Pública 2006; 22:1873-9.

35. Sousa TF, Nahas MV, Silva DAS, Del Duca GF, Peres MA. Fatores associados à obesidade central em adultos de Florianópolis, Santa Catarina: estudo de base populacional. Rev Bras Epidemiol 2011; 14:296-309.

36. Gomes F, Telo DF, Souza HP, Nicolau JC, Halpern A, Serrano Júnior CV. Obesidade e doença arterial coronariana: papel da inflamação vascular. Arq Bras Cardiol 2010; 94:273-9.

37. James SA, Fowler-Brown A, Raghunathan TE, Hoewyk JV. Life-course socioeconomic position and obesity in African American women: the Pitt Country Study. Am J Public Health 2006; 96:554-60.

38. Lopes F. Para além da barreira dos números: desigualdades raciais e saúde. Cad Saúde Pública 2005; 21:1595-601.

39. Silveira EA, Kac G, Barbosa LS. Prevalência e fatores associados à obesidade em idosos residentes em Pelotas, Rio Grande do Sul, Brasil: classificação da obesidade segundo dois pontos de corte do índice de massa corporal. Cad Saúde Pública 2009; 25:1569-77.

40. Schneider HJ, Friedrich N, Klotsche J, Pieper L, Nauck M, John U, et al. The predictive value of different measures of obesity for incident cardiovascular events and mortality. J Clin Endocrinol Metab 2010; 95:1777-85.

41. Florencio TT, Ferreira HS, Cavalcante JC, Stux GR, Sawaya AL. Short stature, abdominal obesity, insulin resistance and alterations in lipid profile in very low income women living in Maceio, northeastern Brazil. Eur J Cardiovasc Prev Rehabil 2007; 14:346-8.

42. Sampaio LR. Avaliação nutricional e envelhecimento. Rev Nutr 2004; 17:507-14.
43. Siqueira VO, Costa BVL, Lopes ACS, Santos LC, Lima-Costa MF, Caiaffa WT. Different equations for determining height among the elderly: the Bambuí cohort study of aging. Cad Saúde Pública 2012; 28:125-34.

44. Monteiro CA, Conde WL, Castro IRR. A tendência cambiante da relação entre escolaridade e risco de obesidade no Brasil (1975-1997). Cad Saúde Pública 2003; 19 Suppl 1:S67-75.

45. Gigante DP, França GVA, Sardinha LMV, Iser BPM, Meléndez GV. Variação temporal na prevalência de sobrepeso e obesidade em adultos: Brasil, 2006 a 2009. Rev Bras Epidemiol 2011; 14 Suppl 1:S157-65.

46. Instituto de Pesquisa Econômica Aplicada. Retrato das desigualdades de gênero e raça. Brasília: Instituto de Pesquisa Econômica Aplicada; 2011.

47. Gigante DP, Moura EC, Sardinha LMV. Prevalência de excesso de peso e obesidade e fatores associados, Brasil, 2006. Rev Saúde Pública 2009; 43 Suppl 2:83-9.

48. Lignani JB, Sichieri R, Burlandy L, Salles-Costa R. Changes in food consumption among the Programa Bolsa Família participant families in Brazil. Public Health Nutr 2011; 14:785-92.

49. Levy-Costa RB, Sichieri R, Pontes NS, Monteiro CA. Disponibilidade domiciliar de alimentos no Brasil: distribuição e evolução (1974-2003). Rev Saúde Pública 2005; 39:530-40.

50. Sá NNB, Moura EC. Excesso de peso: determinantes sócio-demográficos e comportamentais em adultos, Brasil, 2008. Cad Saúde Pública 2011; 27:1380-92.

51. Leventhal AM, Mickens L, Dunton G, Pentz MA, Riggs NR, Sussman S. Tobacco use moderates the association between major depression and obesity. Health Psychol 2010; 29:521-8.

52. Valkengoed IGM, Agyemang C, Krediet RT, Stronks $\mathrm{K}$. Ethnic differences in the association between waist-to-height ratio and albumin-creatinine ratio: the observational SUNSET study. BMC Nephrol 2012; 13:26.

53. Maio MC, Monteiro S, Chor D, Faerstein E, Lopes CS. Cor/raça no Estudo Pró-Saúde: resultados comparativos de dois métodos de autoclassificação no Rio de Janeiro, Brasil. Cad Saúde Pública 2005; 21:171-80.

54. Bastos JL, Peres MA, Peres KG, Dumith SC, Gigante DP. Diferenças socioeconômicas entre autoclassificação e heteroclassificação de cor/raça. Rev Saúde Pública 2008; 42:324-34.

Recebido em 14/Jan/2013

Versão final reapresentada em 25/Jul/2013

Aprovado em 14/Ago/2013 